\title{
Stabilisation of linear PDEs by Stratonovich noise
}

\author{
Tomás Caraballo $^{\mathrm{a}, *}$ and James C. Robinson ${ }^{\mathrm{b}}$ \\ ${ }^{a}$ Dpto. Ecuaciones Diferenciales y Análisis Numérico, Universidad de Sevilla, \\ Apdo. Correos 1160, 41080-Sevilla, Spain. \\ ${ }^{\mathrm{b}}$ Mathematics Institute, University of Warwick, Coventry CV4 7AL, U.K.
}

\begin{abstract}
Some results concerning the stability and stabilisation of stochastic linear partial differential equations in the sense of Stratonovich are proved. The main result ensures that a deterministic linear PDE can be stabilised by adding a suitable Stratonovich noise if and only if the linear partial differential operator has negative trace.
\end{abstract}

Key words: exponential stability, stabilization, Ito's noise, Stratonovich's noise, stochastic PDE.

1991 MSC: 35R10, 35B40, 47H20, 58F39, 73K70

\section{Introduction and statement of the problem}

The use of stochastic partial differential equations in Physics, Chemistry, Biology, Economics, Engineering, etc, is widespread. The addition of random elements (noise) is based on the assumption that such equations are a better model of reality than their deterministic counterparts. Depending on the situation being modelled one can find arguments justifying either of the canonical choices of noise (Itô or Stratonovich). We will not discuss this is detail here, but will emphasise that these different types of noise can produce very different long-time behaviour of solutions (see Caraballo \& Langa [6]).

A fundamental question is the following: assuming that the real world is actually non-deterministic, are deterministic models good approximations? If the answer is affirmative, then the use of such models would be justified, but if the answer is negative, then in some situations the addition of noise could

\footnotetext{
* Corresponding author

Email addresses: caraball@us.es (Tomás Caraballo), jcr@maths.warwick.ac.uk (James C. Robinson).
} 
produce dramatic changes in the behaviour. Here we consider the long-time behaviour of solutions, and investigate the stabilising (or destabilising) effect of the addition of noise.

In the finite-dimensional context, there is a wide literature about such problems (see Arnold [3], Arnold et al. [4], Mao [13], Scheutzow [15], ...). Many results on the stabilisation and destabilisation produced by both Itô and Stratonovich noise have been obtained, and these have also been applied to construct feedback stabilisers (an important task in control problems). Although in each particular situation one or other choice of the noise may be more appropriate, it is stabilisation by Stratonovich noise that is more significant. This is the main motivating reason for this paper, and we now explain this in more detail.

Consider the linear $n$-dimensional ordinary differential equation

$$
\dot{x}=A x,
$$

where $A$ could have unstable directions, and the following stochastic versions of this equation corresponding to the two different interpretations of the stochastic integral

$$
\begin{gathered}
\mathrm{d} x=A x \mathrm{~d} t+\sigma x \circ \mathrm{d} W(t) \quad \text { (Stratonovich) } \\
\mathrm{d} y=A y \mathrm{~d} t+\sigma y \mathrm{~d} W(t), \quad \text { (Ito) }
\end{gathered}
$$

where $W(t)$ is a standard Wiener process on the complete probability space $(\Omega, \mathcal{F}, \mathbb{P})$. The initial value problems for $(2)$ and $(3)$, i.e., to find the solutions of these equations satisfying $x(0)=x_{0}, y(0)=y_{0}$, can be solved explicitly, since their solutions are given by

$$
x(t)=\mathrm{e}^{\sigma W(t)} \exp (t A) x_{0} \quad \text { and } \quad y(t)=\mathrm{e}^{-\frac{\sigma^{2}}{2} t+\sigma W(t)} \exp (t A) y_{0} .
$$

Taking into account the properties of the Wiener process (see e.g. Arnold [1]), it is easy to see that for $\sigma$ large enough the zero solution is exponentially stable for the Itô equation (with probability one), while the same is not true for the Stratonovich equation. This seems to imply that Itô noise has a more profound stabilising effect than Stratonovich noise.

However, this argument is somewhat misleading. Indeed, Eq. (1) can be obviously stabilised by using a simple deterministic feedback control, i.e., the new equation

$$
\dot{x}=A x+\lambda x
$$

becomes exponentially stable provided $\lambda<0$ and $|\lambda|$ is large enough. Similarly, it could be stabilised with the periodic control $\lambda(t) x$,

$$
\dot{x}=A x+\lambda(t) x,
$$

where $\lambda(t)=\lambda_{0}+\sin t$, with $\lambda_{0}<0$ and $\left|\lambda_{0}\right|$ large enough. The function $\sin t$ is, in some sense, a mean-zero function. The same is true with faster meanzero periodic fluctuations: stabilisation takes place because of the systematic 
dissipative term $\lambda_{0} x$ in the equation, while the mean-zero property means that the other term has no influence on the asymptotic behaviour. We can write such an equation in the form

$$
\dot{x}=A x+\lambda_{0} x+x \dot{W}_{\varepsilon}(t)
$$

where $\dot{W}_{\varepsilon}(t)$ denotes the zero mean periodic term and may be considered as a physically realistic approximation of the ideal white noise $\dot{W}(t)$. It is therefore not surprising that the same result is true in the limit when the regular meanzero fluctuations tend to a mean-zero white noise, and the systematic term $\lambda_{0} x$ is still present. Now, it is well known that in such a limit (more precisely in all cases where there are rigorous results concerning the Wong-Zakai [17] approximation of a stochastic equation by a random equation with regularised noise) the correct stochastic interpretation for the equation is the Stratonovich one (see, e.g., Sussmann [16] for a more detailed analysis)

$$
\mathrm{d} x=A x \mathrm{~d} t+\lambda_{0} x \mathrm{~d} t+\sigma x \circ \mathrm{d} W(t)
$$

where $\sigma>0$ describes the intensity of the noise. While it should be intuitively clear that this equation is exponentially stable when $\lambda_{0}<0$ is sufficiently small, a rigorous proof follows from the Itô formula, since as we previously mentioned an explicit form for the solution is

$$
x(t)=\mathrm{e}^{\lambda_{0} t+\sigma W(t)} \exp (A t) x(0) .
$$

Notice that this result is independent of $\sigma$. However, the previous Stratonovich equation can be rewritten in its equivalent Itô form:

$$
\mathrm{d} x=A x \mathrm{~d} t+\lambda_{0} x \mathrm{~d} t+\sigma x \mathrm{~d} W(t)+\frac{\sigma^{2}}{2} x \mathrm{~d} t
$$

If we choose a white noise with intensity such that $\frac{\sigma^{2}}{2}=-\lambda_{0}$, we arrive at the Itô equation

$$
\mathrm{d} x=A x \mathrm{~d} t+\sigma x \mathrm{~d} W(t) .
$$

As a consequence of this easy analysis, it is clear that this equation is exponentially stable with probability one for $\sigma$ large enough.

In general, the "moral" of this example is that Itô equations with multiplicative noise correspond to the limit of deterministic equations with a mean-zero fluctuating control plus a stabilising systematic control. So, the fact that an Itô equation such as (5) is exponentially stable, even if the equation (1) is not, should not be much more surprising than the fact that Eq. (4) with sufficiently large $\lambda<0$ is exponentially stable; it is only that the mathematics required for a proof is more elaborate.

There is a non-trivial literature on stabilisation by Stratonovich noise, with both mathematical and engineering contributions (see [3],[4] and the references therein). Since such a noise is like a periodic zero-mean feedback control, its stabilising effect is unexpected and very intriguing. In the finite-dimensional 
case, Arnold and his collaborators have proved that the linear differential system (1) can be stabilised by the addition of a collection of multiplicative noisy terms,

$$
\mathrm{d} x=A x \mathrm{~d} t+\sum_{i=1}^{d} B_{i} x \circ \mathrm{d} W_{i}(t),
$$

where $W_{i}$ are mutually independent Wiener process and $B_{i}$ are suitable skewsymmetric matrices, if and only if

$$
\operatorname{tr} A<0 .
$$

[Note that the form of the noise is more complex than just a single multiplicative term of the form $\sigma x \circ \mathrm{d} W_{t}$.]

The corresponding problem for linear partial differential equations has been open for a long time, perhaps because one avenue would be to follow a similar approach but in the infinite-dimensional case, e.g. by proving a version of the celebrated Oseledec Multiplicative Ergodic Theorem for infinite-dimensional spaces. In this paper we use a very simple argument to show that one can obtain a similar stabilisation result for a linear PDE $\mathrm{d} u / \mathrm{d} t=A u$ with a finite sum of Stratonovich terms as in (6). Essentially we will reduce the problem to a finite-dimensional one and then apply the result of Arnold et al.

Although our main aim in this paper is to find out conditions under which we can stabilise an unstable linear PDE by using suitable linear Stratonovich noise $^{1}$, our analysis will be more complete. Indeed, we consider a linear evolution equation on a separable Hilbert space $H$ given by

$$
\frac{\mathrm{d} u}{\mathrm{~d} t}=A u,
$$

where $A$ is a linear (unbounded) operator, i.e., $A: D(A) \subset H \rightarrow H$, and consider the stochastically perturbed evolution equation

$$
\mathrm{d} u=A u \mathrm{~d} t+\sum_{i=1}^{N} B_{i} u \circ \mathrm{d} W_{i}
$$

where $B_{i}: D\left(B_{i}\right) \subset H \rightarrow H$ are linear operators and $W_{i}$ are mutually independent Wiener process on the same probability space $(\Omega, \mathcal{F}, \mathbb{P})$.

In Section 2 we first prove that the stability of (8) and (9) are equivalent if the operators $B_{i}$ and $A$ are mutually commuting and satisfy other additional assumptions. It is remarkable that if the noise is considered in the Itô sense it may produce stabilisation or even destabilisation under the same assumptions (see Caraballo \& Langa [6] for a detailed analysis). Section 3 gives some sufficient conditions that ensure the stability of the deterministic equation (8)

1 The reader is referred to Caraballo et al. [7], [5], Caraballo \& Langa [6], Kwiecinska [11], Leha et al. [12] for similar results but using noisy terms in the sense of Itô. 
is transferred to the stochastic equation (9) when the operator $A$ does not commute with some $B_{i}$. Finally, in Section 4 , we will prove that (9) becomes exponentially stable with probability 1 for suitable operators $B_{i}$ if and only if the trace of $A$ is negative, an infinite-dimensional analogue of the results of Arnold et al.

\section{Stability of stochastic PDEs: the commutative case}

In this section we will establish a result concerning the exponential stability of the null solution to a linear stochastic PDE. Roughly, we will show that the presence of linear (unbounded) operators in the diffusion part do not modify the exponential stability of the null solution provided that they commute with the linear operator in the drift.

To start with, we will consider the problem in the Itô formulation, so that we can apply a result due to Da Prato \& Zabczyk [8] which ensures the equivalence of the stochastic PDE to a nonautonomous deterministic one depending on a random parameter, i.e. a random PDE. Then, we will transform our Stratonovich model into its equivalent ${ }^{2}$ Itô one and will apply this result.

Let us consider

$$
\left\{\begin{array}{l}
\mathrm{d} u=A u \mathrm{~d} t+\sum_{k=1}^{d} B_{k} u \mathrm{~d} W_{k}, \\
u(0)=u_{0} \in H .
\end{array}\right.
$$

where $A: D(A) \subset H \rightarrow H, B_{k}: D\left(B_{k}\right) \subset H \rightarrow H, k=1,2, \cdots, d$ are generators of $C_{0}$-semigroups $S_{A}(t)$ and $S_{k}$ respectively, and $W_{1}, \cdots, W_{d}$ are independent real Wiener processes.

We will need the following additional assumptions.

The operators $B_{1}, \cdots, B_{d}$ generate mutually commuting $C_{0}-$ groups $S_{k}$

$$
D\left(B_{k}^{2}\right) \supset D(A) \text { for } k=1, \cdots, d \text { and } \bigcap_{k=1}^{d} D\left(\left(B_{k}^{*}\right)^{2}\right) \text { is dense in } H,
$$

where $B_{k}^{*}$ denotes the adjoint operator of $B_{k}$;

$$
C=A-\frac{1}{2} \sum_{k=1}^{d} B_{k}^{2} \quad \text { generates a } C_{0}-\text { semigroup } S_{C}
$$

[Note that $D(C)=D(A)$.]

\footnotetext{
2 This equivalence has been proved by Kunita [10] for suitable partial differential operators. We implicitly assume that we are considering this case. It is undoubtedly an important task to develop a general theory of stochastic PDEs in the Stratonovich sense.
} 
Now, given a fixed realisation of our Wiener processes $W_{k}(t, \omega), \omega \in \Omega$, in order to solve (10) we define

$$
U_{\omega}(t)=\prod_{k=1}^{d} S_{k}\left(W_{k}(t, \omega)\right) \text { and } v(t)=U_{\omega}^{-1}(t) u(t), \quad t \geq 0,
$$

and consider the equation

$$
\left\{\begin{array}{l}
\dot{v}(t)=U_{\omega}^{-1}(t) C U_{\omega}(t) v(t) \\
v(0)=u_{0}
\end{array}\right.
$$

which is a deterministic equation depending on the parameter $\omega$. The following result, along with the definition of a strong solution, can be found in Da Prato and Zabczyk [8]

Proposition 2.1 Assume conditions (11)-(13). If $u$ is a strong solution to (10), then the process $v(t, \omega)$ defined by (14) satisfies (15). Conversely, if $v$ is a predictable process whose trajectories are continuously differentiable and satisfy $(15) \mathbb{P}$-a.s., then the process $u(t, \omega)=U_{\omega}(t) v(t, \omega)$ takes values in $D(C)$ $\mathbb{P}$-a.s and for almost all $t$, and is a strong solution to (10).

Remark 2.2 One can also find in Da Prato \& Zabczyk [8] a sufficient condition ensuring solvability of (15) which could be useful in applications (see [8, pp. 177-179] for more details).

Now, we consider our original Stratonovich version of the problem,

$$
\left\{\begin{array}{l}
\mathrm{d} u=A u \mathrm{~d} t+\sum_{k=1}^{d} B_{k} u \circ \mathrm{d} W_{k}, \\
u(0)=u_{0} \in H
\end{array}\right.
$$

To ensure existence of solutions to this problem, we can consider its equivalent Itô version

$$
\left\{\begin{array}{l}
\mathrm{d} u=\left(A+\frac{1}{2} \sum_{k=1}^{d} B_{k}^{2}\right) u \mathrm{~d} t+\sum_{k=1}^{d} B_{k} u \mathrm{~d} W_{k}, \\
u(0)=u_{0} \in H
\end{array}\right.
$$

If we now assume conditions (11), (12), and the following (18) instead of (13),

$$
C=A+\frac{1}{2} \sum_{k=1}^{d} B_{k}^{2}, \quad \text { generates a } C_{0}-\operatorname{semigroup} S_{C},
$$

[we still have $D(C)=D(A)$ ] we can ensure (thanks to Proposition 2.1) that our problem (16) can be written in an equivalent way as

$$
\left\{\begin{array}{l}
\dot{v}(t)=U_{\omega}^{-1}(t) A U_{\omega}(t) v(t) \\
v(0)=u_{0}
\end{array}\right.
$$

We now prove the following result which, in particular, generalises a similar one in Caraballo and Langa [6]. 
Theorem 2.3 In addition to assumptions (11), (12), and (18), suppose that $A$ commutes with each $S_{k}(t)$. Then the strongly continuous semigroup $S_{A}(t)$ generated by $A$ is exponentially stable, i.e. there exist $M_{0}, \gamma>0$ such that $\left|S_{A}(t)\right| \leq M_{0} e^{-\gamma t}$ for all $t>0$, if and only if there exist $\alpha, C>0$ and $\Omega_{0} \subset \Omega$ with $P\left(\Omega_{0}\right)=0$ such that for $\omega \notin \Omega_{0}$ there exists $T(\omega)>0$ such that the following holds for the solution of (16),

$$
|u(t)| \leq C\left|u_{0}\right| e^{-\alpha t} \quad \text { for } t \geq T(\omega) \text { almost surely. }
$$

Proof. $\Rightarrow$ Let us denote by $u(t)=u\left(t, \omega ; 0, u_{0}\right)$ the solution of (16) for $u_{0} \in D(A)$, and by $v(t)=v\left(t, \omega ; 0, u_{0}\right)$ the corresponding solution to (19), i.e. $v(t)=U_{\omega}^{-1}(t) u(t)$. But, owing to the commutativity of operator $A$ and operators $S_{k}$, the problem (19) can be written now as

$$
\left\{\begin{array}{l}
\dot{v}(t)=A v(t) \\
v(0)=u_{0} \in D(A),
\end{array}\right.
$$

whose solution is given by $v(t)=S_{A}(t) u_{0}$, so we have an explicit expression for our solution $u(t)$ :

$$
u(t)=u\left(t, \omega ; 0, u_{0}\right)=U_{\omega}(t) S_{A}(t) u_{0} .
$$

Now, since we know that $S_{A}(t)$ is exponentially stable, there exist $M_{0}, \gamma>0$ such that $|S(t)| \leq M_{0} \mathrm{e}^{-\gamma t}$ for all $t>0$. It is straightforward to bound $|u(t)|$ as follows. First, we notice that (see e.g. Pazy [14]) there exists $M_{k}>0, b_{k} \in \mathbb{R}$ such that $\left|S_{k}(t)\right| \leq M_{k} e^{b_{k}|t|}, k=1, \cdots, d$. Thus,

$$
\begin{aligned}
|u(t)| & \leq\left|U_{\omega}(t) S_{A}(t) u_{0}\right| \\
& \leq\left|U_{\omega}(t)\right|\left|S_{A}(t)\right|\left|u_{0}\right| \\
& \leq M M_{1} \cdots M_{d}\left|u_{0}\right| \exp \left\{-\gamma t+\sum_{k=1}^{d} b_{k}\left|W_{k}(t)\right|\right\} \\
& \leq C\left|u_{0}\right| \exp \left\{t\left(-\gamma+\sum_{k=1}^{d} \frac{b_{k}\left|W_{k}(t)\right|}{t}\right)\right\}
\end{aligned}
$$

and as

$$
\lim _{t \rightarrow+\infty} \frac{\left|W_{k}(t, \omega)\right|}{t}=0, \text { for all } k=1, \cdots, d,
$$

there exists $\Omega_{0} \subset \Omega$ with $\mathbb{P}\left(\Omega_{0}\right)=0$ such that if $\omega \notin \Omega_{0}$, then

$$
\lim _{t \rightarrow+\infty}\left(\gamma-\sum_{k=1}^{d} \frac{b_{k}\left|W_{k}(t, \omega)\right|}{t}\right)=\gamma
$$

and, there exists $T(\omega)$ such that for all $t \geq T(\omega)$

$$
\gamma-\sum_{k=1}^{d} \frac{b_{k}\left|W_{k}(t, \omega)\right|}{t} \geq \frac{\gamma}{2} .
$$


Thus, this part of the proof is complete if we set $\alpha=\gamma / 2$. If we now consider $u_{0} \in H$ we can argue by approximation since $D(A)$ in dense in $H$.

$\Leftarrow$ We omit the converse since the proof is similar taking into account now that $v(t)=U_{\omega}^{-1}(t) u(t)$, the properties of strongly continuous semigroups and the Wiener processes.

\section{Stability of stochastic PDEs: the noncommutative case}

Notice that under our commutativity assumptions in the previous section, the deterministic problem is exponentially stable if and only if the stochastically perturbed equation has the same property. However, an immediate question arises. What happens if no commutativity holds? In this case we will be able to prove some sufficient conditions for the exponential stability of the zero solution.

Theorem 3.1 Assume that (11),(18) hold, and there exists $\gamma>0$ such that $\left|S_{A}(t)\right| \leq e^{-\gamma t}$ for all $t \geq 0$. If in addition we assume that $U_{\omega}(t)$ is unitary for all $t \geq 0$ and almost all $\omega$ (i.e., $U_{\omega}^{*}(t)=U_{\omega}^{-1}(t)$ ) then, there exists $\alpha>0$ such that the following holds for the solution of (16),

$$
|u(t)| \leq\left|u_{0}\right| e^{-\alpha t}
$$

for $t \geq 0$ almost surely.

Proof. First, we notice that an operator $A$ generates a strongly continuous semigroup $S_{A}(t)$ satisfying $\left|S_{A}(t)\right| \leq \mathrm{e}^{\alpha t}, \alpha \in \mathbb{R}$, if and only if $(A u, u) \leq \alpha|u|^{2}$, for all $u \in D(A)$. We can now consider our problem

$$
\left\{\begin{array}{l}
\dot{v}(t)=U_{\omega}^{-1}(t) A U_{\omega}(t) v(t) \\
v(0)=u_{0} \in D(A)
\end{array}\right.
$$

and observe that, thanks to our assumptions,

$$
\begin{aligned}
\frac{\mathrm{d}}{\mathrm{d} t}|v(t)|^{2} & =2\left(U_{\omega}^{-1}(t) A U_{\omega}(t) v(t), v(t)\right) \\
& \leq 2\left(A U_{\omega}(t) v(t), U_{\omega}(t) v(t)\right) \\
& \leq-2 \gamma\left|U_{\omega}(t) v(t)\right|^{2} \\
& =-2 \gamma|v(t)|^{2}
\end{aligned}
$$

This implies that

$$
|v(t)|^{2} \leq|v(0)|^{2} \mathrm{e}^{-2 \gamma t}, \text { for all } t \geq 0,
$$

and as $v(t)=U_{\omega}^{-1}(t) u(t)$, we immediately obtain $|u(t)|=|v(t)| \leq\left|u_{0}\right| e^{-\gamma t}$ for $u_{0} \in D(A)$ and all $t \geq 0$. Finally, the result holds true for $u_{0} \in H$ by an approximation argument. 
Nevertheless, when the operator $U$ is not unitary, we can still prove a sufficient condition ensuring this exponential behaviour of solutions. Notice that we do not need the mutual commutativity of the operators $B_{k}$.

Theorem 3.2 Assume that $A$ generates a strongly continuous semigroup $S_{A}(t)$ such that $|S(t)| \leq e^{-\gamma t}$, for all $t \geq 0$ and some $\gamma>0$. Assume that $B_{k}: H \rightarrow H$ is a linear and bounded operator for $k=1, \cdots, d$ such that there exists $b_{k} \geq 0$ satisfying

$$
b_{k}|u|^{2} \leq\left(B_{k} u, u\right), \quad \forall 1 \leq k \leq d, u \in H .
$$

Then for every initial value $u_{0} \in D(A)$ such that $u_{0} \neq 0$, the corresponding solution to (16), $u(\cdot)=u\left(\cdot, \omega ; 0, u_{0}\right)$, is defined in the future and satisfies $|u(t)|>0$ for all $t>0$ almost surely,

$$
\limsup _{t \rightarrow+\infty} \frac{1}{t} \log |u(t)| \leq-\left(\gamma-\sum_{k=1}^{d}\left(\left|B_{k}\right|^{2}-b_{k}^{2}\right)\right) \quad \mathbb{P}-\text { a.s. }
$$

Proof. Take $u_{0} \in D(A)$ such that $u_{0} \neq 0$, then it follows from the theory of linear stochastic partial differential equations that the solution $u(t)$ to (16) (which in addition has a continuous version) is defined in the future and $|u(t)|>0$ for all $t>0, \mathbb{P}-$ a.s. (see Da Prato \& Zabczyk [8] p. 190-191). Then, in order to apply Itô's formula, we consider the equivalent Itô formulation for our problem (17), i.e.

$$
u(t)=u_{0}+\int_{0}^{t}\left(A u(s)+\frac{1}{2} \sum_{k=1}^{d} B_{k}^{2} u(s)\right) \mathrm{d} s+\sum_{k=1}^{d} \int_{0}^{t} B_{k} u(s) \mathrm{d} W_{k}(s) .
$$

Thus, it follows (we omit the argument $s$ in the integrals for short)

$$
\begin{aligned}
& \log |u(t)|^{2} \\
& \leq \log \left|u_{0}\right|^{2}+\int_{0}^{t} \frac{2\left(u, A u+\frac{1}{2} \sum_{k=1}^{d} B_{k}^{2} u\right)+\sum_{k=1}^{d}\left|B_{k} u\right|^{2}}{|u|^{2}} \mathrm{~d} s \\
& \quad-\frac{1}{2} \sum_{k=1}^{d} \int_{0}^{t} \frac{4\left(u, B_{k} u\right)^{2}}{|u|^{4}} \mathrm{~d} s+M(t), \\
& \leq \log \left|u_{0}\right|^{2}+\int_{0}^{t} \frac{2\left((u, A u)+\sum_{k=1}^{d}\left(\frac{1}{2}\left(u, B_{k}^{2} u\right)+\frac{1}{2}\left|B_{k} u\right|^{2}-b_{k}^{2}|u|^{2}\right)\right)}{|u|^{2}} \mathrm{~d} s \\
& \quad+M(t) \\
& \leq \log \left|u_{0}\right|^{2}+2 \int_{0}^{t}\left(-\gamma+\sum_{k=1}^{d}\left(\left|B_{k}\right|^{2}-b_{k}^{2}\right)\right) \mathrm{d} s+M(t)
\end{aligned}
$$

where $M(t)$, owing to our assumptions, is a continuous local martingale given by

$$
M(t)=2 \sum_{k=1}^{d} \int_{0}^{t} \frac{\left(u(s), B_{k} u(s)\right)}{|u(s)|^{2}} \mathrm{~d} W_{k}(s),
$$


and, by means of the law of iterated logarithm, satisfies

$$
\limsup _{t \rightarrow+\infty} \frac{M(t)}{t}=0
$$

Dividing both sides of (24) by $t$ and taking limsup we complete the proof.

Observe that, in this case, we need that the diffusion term be small with respect to the drift to ensure exponential stability. In the particular case in which $\left|B_{k}\right|^{2}=b_{k}^{2}$ (what happens, for instance when $B_{k}$ is given by $B_{k} u=b_{k} u$ ), the exponential decay rate is the same since here $\left|B_{k}\right|^{2}-b_{k}^{2}=0$.

\section{Stabilisation of deterministic PDEs}

Now, we will prove that the negative trace assumption (7) (which will be stated more precisely later on) is a necessary and sufficient condition for the stabilisation of a linear PDE by using a suitable Stratonovich noise.

In order to motivate our general argument we consider the following simple example, a one-dimensional heat equation

$$
\left\{\begin{array}{l}
\frac{\partial u(t, x)}{\partial t}=\frac{\partial^{2} u(t, x)}{\partial x^{2}}+2 u(t, x), t>0,0<x<\pi \\
u(t, 0)=u(t, \pi)=0, t>0 \\
u(0, x)=u_{0}(x), x \in[0, \pi] .
\end{array}\right.
$$

This problem can be formulated in our framework by setting $H=L^{2}([0, \pi])$, $A=\frac{\partial^{2}}{\partial x^{2}}+2 I$; it follows that $D(A)=H_{0}^{1}([0, \pi]) \cap H^{2}([0, \pi])$. It is well known that this problem can be solved explicitly yielding

$$
u(t, x)=\sum_{n=1}^{\infty} a_{n} e^{-\left(n^{2}-2\right) t} \sin n x
$$

where $u_{0}(x)=\sum_{n=1}^{\infty} a_{n} \sin n x$. Hence, it is clear that the zero solution of our problem (25) is not stable. But we will choose an appropriate operator $B: H \rightarrow H$ such that

$$
\left\{\begin{array}{l}
\mathrm{d} u(t, x)=A u(t, x) \mathrm{d} t+B u(t, x) \circ \mathrm{d} W(t) \\
u(0, x)=u_{0}(x)
\end{array}\right.
$$

becomes exponentially stable with probability one. It is worth pointing out that the operator $B$ cannot commute with $A$ if we wish to obtain such a stabilisation result.

Notice that operator $A$ has a sequence of eigenvalues given by $\lambda_{n}=2-$ $n^{2}, n \geq 1$, with corresponding eigenfuntions $e_{n}=\sqrt{\frac{2}{\pi}} \sin n x$, which form an 
orthonormal basis of the Hilbert space $H$. This means that any $u \in H$ can be represented as

$$
u=\sum_{k \geq 1}\left(u, e_{k}\right) e_{k}=\sum_{k \geq 1} u_{k} e_{k}
$$

Now we define $B: H \rightarrow H$ as $B e_{1}=-\sigma e_{2}, B e_{2}=\sigma e_{1}, B e_{n}=0, n \geq 3$ which is a linear operator (and does not commute with $A$ ). Then, using the Fourier representation for the solution $u(t)$ to $(26)$, our problem can be re-written as

$$
\left\{\begin{array}{l}
\sum_{k \geq 1} \mathrm{~d} u_{k}(t) e_{k}=\sum_{k \geq 1} \lambda_{k} u_{k}(t) e_{k} \mathrm{~d} t+\left(\sigma u_{2}(t) e_{1}-\sigma u_{1}(t) e_{2}\right) \circ \mathrm{d} W(t) \\
u(0)=u_{0}=\sum_{k \geq 1} u_{0 k} e_{k} .
\end{array}\right.
$$

Identifying the coefficients we get two coupled problems (the first a 2-dimensional stochastic ordinary differential system, and the second an infinite-dimensional one which is exponentially stable since $\lambda_{n}<0$ for all $n \geq 3$ ):

$$
\left\{\begin{array}{l}
\left(\begin{array}{l}
\mathrm{d} u_{1}(t) \\
\mathrm{d} u_{2}(t)
\end{array}\right)=\left(\begin{array}{cc}
\lambda_{1} & 0 \\
0 & \lambda_{2}
\end{array}\right)\left(\begin{array}{l}
u_{1}(t) \\
u_{2}(t)
\end{array}\right)+\left(\begin{array}{cc}
0 & \sigma \\
-\sigma & 0
\end{array}\right)\left(\begin{array}{l}
u_{1}(t) \\
u_{2}(t)
\end{array}\right) \circ \mathrm{d} W(t) \\
\left(\begin{array}{l}
u_{1}(0) \\
u_{2}(0)
\end{array}\right)=\left(\begin{array}{l}
u_{01} \\
u_{02}
\end{array}\right)
\end{array}\right.
$$

and

$$
\left\{\begin{array}{l}
\sum_{k \geq 3} \mathrm{~d} u_{k}(t) e_{k}=\sum_{k \geq 1} \lambda_{k} u_{k}(t) e_{k} \mathrm{~d} t \\
\sum_{k \geq 3} u_{k}(0) e_{k}=\sum_{k \geq 3} u_{0 k} e_{k}
\end{array}\right.
$$

Now, as the matrix $\left(\begin{array}{cc}0 & 1 \\ -1 & 0\end{array}\right)$ is a basis for the linear space of skew symmetric $2 \times 2$ matrices, Arnold et al. proved in [4] that the leading Lyapunov exponent of solutions to (28) tends to $\left(\lambda_{1}+\lambda_{2}\right) / 2=-1 / 2$ as the parameter $\sigma$ grows to $+\infty$. As it easily follows that the leading Lyapunov exponent for the solutions to (29) is $\lambda_{3}=-7$, we can ensure that the top Lyapunov exponent for the solutions of (27) is negative.

As can be seen in this example, the main idea for the stabilisation is to decompose the problem into two new problems: a finite-dimensional one which can be stabilised by using previously available methods, and another infinitedimensional problem which is already exponentially stable. This idea can be extended in a general way to solve the stabilisation problem for a class of deterministic PDEs which appears very often in applications.

Consider the infinite-dimensional linear system

$$
\dot{u}=A u
$$

where $A: D(A) \subset H \rightarrow H$ is a linear operator which has a sequence of eigenvalues $\lambda_{j}$ with corresponding eigenfunctions $e_{j}$. We assume that these 
eigenfunctions form an orthonormal basis of the separable Hilbert space $H$, and that the eigenvalues $\lambda_{j}$ are bounded above (but not necessarily below), so that they can be ordered $\lambda_{1} \geq \lambda_{2} \geq \ldots$.We denote by $|\cdot|$ the norm in $H$ and by $(\cdot, \cdot)$ its associated scalar product.

Now, we can prove our main stabilisation result.

Theorem 4.1 Assume that the trace of $A$ is negative, in other words that

$$
\sum_{j=1}^{\infty} \lambda_{j}<0
$$

Then there exist linear operators $B_{k}: H \rightarrow H, k=1,2, \ldots, d$, such that for

$$
\mathrm{d} u=A u \mathrm{~d} t+\sum_{j=1}^{d} B_{k} u \circ \mathrm{d} W_{k}
$$

the zero solution is exponentially stable with probability one. The operators $B_{k}$ are such that for some $N>0$, the $N \times N$ matrices $D_{1}, \ldots, D_{k}$ defined as

$$
D_{k}=\left(\begin{array}{cccc}
\left(B_{k} e_{1}, e_{1}\right) & \left(B_{k} e_{2}, e_{1}\right) & \cdots & \left(B_{k} e_{N}, e_{1}\right) \\
\left(B_{k} e_{1}, e_{2}\right) & \left(B_{k} e_{2}, e_{2}\right) & \cdots & \left(B_{k} e_{N}, e_{2}\right) \\
\vdots & \vdots & & \vdots \\
\left(B_{k} e_{1}, e_{N}\right) & \left(B_{k} e_{2}, e_{N}\right) & \cdots & \left(B_{k} e_{N}, e_{N}\right)
\end{array}\right)
$$

are skew-symmetric.

Conversely, if there exist linear operators $B_{k}: H \rightarrow H, k=1,2, \ldots, d$ with the above properties for which the zero solution of (32) is exponentially stable with probability one then the trace of $A$ is negative, i.e. (31) holds.

Proof. Assumption (31) implies, in particular, that there exists an $N \in \mathbb{N}$ such that

$$
\sum_{j=1}^{N} \lambda_{j}<0
$$

Let $P: H \rightarrow P H$ be the projection onto the first $N$ eigenfunctions, and $Q$ its orthogonal complement, i.e, given $u \in H$ we have

$$
P u=\sum_{j=1}^{N}\left(u, e_{j}\right) e_{j}, \quad Q u=\sum_{j=N+1}^{\infty}\left(u, e_{j}\right) e_{j} .
$$

Write $p=P u$ and $q=Q u$, and let

$$
A_{-}=P A P \quad \text { and } \quad A_{+}=Q A Q
$$

In particular, $A_{-}$corresponds to a finite-dimensional $(N \times N)$ matrix with negative trace, while $A_{+}$is a negative definite operator since it easily follows that $\left(A_{+} u, u\right) \leq \lambda_{N+1}|u|^{2}$ for any $u \in H$. 
Our original deterministic partial differential equation (30) is equivalent to the coupled system

$$
\begin{aligned}
& \dot{p}=A_{-} p \\
& \dot{q}=A_{+} q,
\end{aligned}
$$

so that all the possible 'unstable' behaviour occurs in the finite-dimensional $p$ equation, while the origin is stable for the $q$ equation. Since the trace of $A_{-}$is negative, this part can be stabilised thanks to the finite-dimensional results by Arnold and his collaborators (see [3] and [4]), i.e. there exist $N \times N$ skew-symmetric matrices $C^{(k)}$ such that the stochastic ordinary differential system

$$
\mathrm{d} p=A_{-} p \mathrm{~d} t+\sum_{k=1}^{d} C^{(k)} p \circ \mathrm{d} W_{k}
$$

is exponentially stable with probability one. Now we define operators $M_{k}$ : $H \rightarrow H$ as follows: if we assume that $u=\sum_{k \geq 1} u_{k} e_{k}$ and $C^{(k)}=\left(\left(c_{i j}^{(k)}\right)\right)_{1 \leq i, j \leq N}$, and we set

$$
B_{k} u=\sum_{i=1}^{N}\left(\sum_{j=1}^{N} c_{i j}^{(k)} u_{j}\right) e_{i},
$$

then it follows that the equation

$$
\mathrm{d} u=A u \mathrm{~d} t+\sum_{k=1}^{d} B_{k} u \circ \mathrm{d} W_{k}
$$

is now stable.

Conversely, if there exist operators $B_{k}, k=1,2, \ldots, d$, such that (32) is pathwise exponentially stable, then it is easy to check that for any $r \in \mathbb{N}$, the corresponding finite dimensional system in $\mathbb{R}^{r}$

$$
\mathrm{d} p_{r}=A_{-} p_{r} \mathrm{~d} t+\sum_{k=1}^{d} D_{k} p_{r} \circ \mathrm{d} W_{k}
$$

is also exponentially stable with probability one: indeed, $\left|u(t, \omega) u_{0}\right| \leq C e^{-\gamma t}\left|u_{0}\right|$ implies that $\left|P_{r} u(t, \omega) u_{0}\right| \leq C e^{-\gamma t}\left|u_{0}\right|$, and therefore the solutions to (34) decay to zero exponentially fast and almost surely. Now we claim that there exists $r \in \mathbb{N}$ such that

$$
\sum_{i=1}^{r} \lambda_{i}<0
$$

If not, i.e., if for any $r \in \mathbb{N}$ we have that $\sum_{i=1}^{r} \lambda_{i} \geq 0$, then, in particular we have $\sum_{i=1}^{N} \lambda_{i} \geq 0$, contradicting the result of Arnold et al. since the ordinary differential system $\mathrm{d} p_{N}=A_{-} p_{N} \mathrm{~d} t$ would have been stabilised by using a finite number of skew-symmetric matrices despite the fact that the trace of the matrix $A_{-}$is non-negative. So $\sum_{i=1}^{N} \lambda_{i}$ is negative and consequently $\sum_{i=1}^{\infty} \lambda_{i}$ is negative too.

\section{Conclusions}


In this paper we have characterised when a wide class of linear PDEs can be stabilised by using a suitable Stratonovich noise. We have also indicated why this is more significant than the similar effect produced by Itô noise, which really only reflects the systematic linear stabilisation inherent in such terms. However, we should remark here that noise can also produce destabilisation (see Arnold [2] pp. 162 for more details).

Acknowledgements. This work was started while the first author was visiting the Mathematics Institute (University of Warwick) in the summer 2002 within the Programa de Movilidad del Profesorado Universitario (Ministerio de Educación y Cultura, Spain) and a joint project grant funded by the Royal Society. He wishes express his thanks to all the people in the Institute for the kind hospitality they offered him, especially to James Robinson who, jointly with his wife Tania Styles, made him and his family enjoy a lovely stay in UK. JCR is a Royal Society University Research Fellow, and would like to thank the Society for all their support.

\section{References}

[1] L. Arnold, Stochastic Differential Equations: Theory and Applications, Wiley \& Sons, New York, (1974).

[2] L. Arnold, Random Dynamical Systems. Springer, New York, 1998.

[3] L. Arnold, Stabilization by noise revisited, Z. Angew. Math. Mech. 70(1990), $235-246$.

[4] L. Arnold, H. Crauel \& V. Wihstutz, Stabilization of linear systems by noise, SIAM J. Control Optim. 21(1983), 451-461.

[5] T. Caraballo, M.J. Garrido-Atienza \& J. Real, Stochastic stabilization of differential systems with general decay rate, Systems \& Control Letters 48(5) (2003), 397-406.

[6] T. Caraballo \& J.A. Langa, Comparison of the long-time behavior of linear Ito and Stratonovich partial differential equations, Stoch. Anal. Appl. 19(2) (2001), 183-195.

[7] T. Caraballo, K. Liu \& X.R. Mao, On stabilization of partial differential equations by noise, Nagoya Math. J. 161(2) (2001), 155-170.

[8] G. Da Prato \& J. Zabczyk, Stochastic Equations in Infinite Dimensions, Cambridge University Press, (1992).

[9] R. Has'minskiI, Stochastic Stability of Differential Equations, Sijthoff and Noordhoff, Netherlands, (1980).

[10] H. Kunita, Stochastic Partial Differential Equations connected with NonLinear Filtering, in Lecture Notes in Mathematics 972, pp. 100-169, (1981)

[11] A.A. Kwiecinska, Stabilization of evolution equations by noise, Proc. Amer. Math. Soc. 130(2002), No. 10, 3067-3074. 
[12] G. Leha, B. Maslowski \& G. Ritter, Stability of solutions to semilinear stochastic evolution equations, Stoch. Anal. Appl. 17(1999), No. 6, 1009-1051.

[13] X.R. Mao, Stochastic stabilization and destabilization, Systems \& Control Letters 23 (1994), 279-290.

[14] A. PAZY, Semigroups of Linear Operators and Applications to Partial Differential Equations, Springer-Verlag, New York Inc., 1983.

[15] M. Scheutzow, Stabilization and destabilization by noise in the plane, Stoch. Anal. Appl. 11(1) (1993), 97-113.

[16] H.J. Sussmann, On the gap between deterministic and stochatic ordinary differential equations, The Annals of Probability 6(1978), No. 1, 19-41.

[17] E. Wong \& M. ZAKAI, On the relationship between ordinary and stochastic differential equations and applications to stochastic problems in control theory, Proc. Third IFAC Congress, paper 3B, 1966. 